\title{
The supremum of random walk with negatively associated and heavy-tailed steps
}

\author{
Dingcheng Wang ${ }^{\mathrm{a}, \mathrm{b}, *}$, Pingyan $\mathrm{Chen}^{\mathrm{c}}$, Chun $\mathrm{Su}^{\mathrm{d}}$ \\ ${ }^{a}$ Center of Financial Mathematics, MSI, Australian National University, Canberra, Act 0200, Australia \\ ${ }^{\mathrm{b}}$ School of Applied Mathematics and School of Management, University of Electronic Science and Technology of China, \\ Chengdu 610054, Sichuan, P.R. China \\ ${ }^{\mathrm{c}}$ Department of Mathematics, Jinan University, Guangzhou 510630, P.R. China \\ ${ }^{\mathrm{d}}$ Department of Statistics and Finance, University of Science and Technology of China, Hefei 230026, Anhui, P.R. China
}

Received 14 June 2006; received in revised form 19 December 2006; accepted 21 February 2007

Available online 7 April 2007

\begin{abstract}
This paper obtains the Embrechts-Veraverbeke asymptotic formula for the random walk with dependent steps, where the steps constitute a sequence of negatively associated random variables with a common heavy-tailed distribution such that its left tail is lighter than its right tail.
\end{abstract}

(C) 2007 Elsevier B.V. All rights reserved.

Keywords: Equilibrium distribution; Heavy-tails; Negative association; Random walk; Supremum

\section{Introduction}

\subsection{Background}

Throughout, $C$ represents an absolute positive constant, which may vary from different places. Denote $a^{+}=\max \{a, 0\}$ and $a^{-}=\min \{-a, 0\}$. For two positive infinitesimals $A(\cdot)$ and $B(\cdot)$, we write $A \lesssim B$ if lim sup $A / B \leqslant 1$ and $A \gtrsim B$ if $\lim \inf A / B \geqslant 1$; we write $A \sim B$, as usual, if both the relations hold; we also write $A=\mathrm{o}(B)$ if $\lim A / B=0$. In the sequel, all limit relations are for $x \rightarrow \infty$ unless stated otherwise.

For a random variable (r.v.) $X$ with distribution function (d.f.) $F$ concentrated on $(-\infty, \infty)$, we denote its tail by $\bar{F}(x)=1-F(x)=\mathbb{P}(X>x)$ and write $F^{+}(x)=F(x) \rrbracket_{(0 \leqslant x<\infty)}$, where $\rrbracket_{A}$ denotes the indicator function of event $A$. Clearly, $F^{+}$is the d.f. of r.v. $X^{+}$. For a d.f. $F$ concentrated on $(-\infty, \infty)$ with a finite mean and

\footnotetext{
${ }^{27}$ Wang's work was supported by MASCOS grant from Australian Research Council and National Natural Science Foundation of China (Grant no. 70671018). Chen's work was supported by National Natural Science Foundation of China (Grant no. 60574002).

*Corresponding author. Center of Financial Mathematics, MSI, Australian National University, Canberra, Act 0200, Australia.

E-mail addresses: Dingcheng.Wang@maths.anu.edu.au, wangdc@uestc.edu.cn (D.C. Wang), chenpingyan@263.net (P.Y. Chen), suchun@ustc.edu.cn (C. Su).
} 
$v=\int_{0}^{\infty} \bar{F}(t) \mathrm{d} t>0$, we also denote its equilibrium distribution by

$$
F_{\mathrm{e}}(x)=\frac{1}{v} \int_{0}^{x} \bar{F}(t) \mathrm{d} t, \quad x \geqslant 0 .
$$

Clearly, $F_{\mathrm{e}}$ is also the equilibrium distribution of $F^{+}$.

The standing assumption of this work is that the common d.f. $F$ is heavy tailed. The most important class of heavy-tailed distributions is the subexponential class. By definition, $F$ concentrated on $[0, \infty)$ is said to be subexponential, denoted by $\mathscr{S}$, if

$$
\lim _{x \rightarrow \infty} \frac{\bar{F}^{* n}(x)}{n \bar{F}(x)}=1
$$

holds for any (or equivalently, for some ) $n \geqslant 2$, where $F^{* n}$ denotes the convolution of $F$. More generally, $F$ concentrated on $(-\infty, \infty)$ is said to be subexponential if $F^{+}$is subexponential. Closely related are the class $\mathscr{D}$ of distributions with dominated variations and the class $\mathscr{C}$ with consistently varying tails. By definition, $F \in \mathscr{D}$ if

$$
\limsup _{x \rightarrow \infty} \frac{\bar{F}(l x)}{\bar{F}(x)}<\infty
$$

holds for any $0<l<1$ (or equivalently, for some $0<l<1$ ); $F \in \mathscr{C}$ if

$$
\lim _{l \nearrow 1} \limsup _{x \rightarrow \infty} \frac{\bar{F}(l x)}{\bar{F}(x)}=1 \quad \text { or equivalently } \quad \lim _{l \searrow 1} \liminf _{x \rightarrow \infty} \frac{\bar{F}(l x)}{\bar{F}(x)}=1 .
$$

The regular property in (1.3) of the tail probability $\bar{F}$ was first introduced and named as "intermediate regular varying property" by Bingham et al. (1987). It is well known that

$$
\mathscr{C} \subset \mathscr{D} \text { and } \mathscr{C} \subset \mathscr{S} \text {. }
$$

The class $\mathscr{C}$ is a useful class of heavy-tailed distributions. Specially, it contains the famous class $\mathscr{R}$ of distributions with regular variations and a slightly larger class, the class of distributions with extended regular variations (ERV).

In various fields such as insurance mathematics, queuing theory, finance and time series analysis, the model of a random walk with negative drift occurs in natural ways, for example, infinite-horizon ruin probability can be viewed as the tail probability of the supremum of a random walk with negative drift. There exists an extensive literature on the asymptotic behavior of the supremum of a random walk. Most of the literature deals with the random walk with independent and identically distributed (i.i.d.) steps for many different kinds of subclasses of heavily tailed distributions. We refer the reader to Embrechts et al. (1997) for the most important results and additional references.

A classical result for the asymptotic behavior is from Embrechts-Veraverbeke (1982), which tells us that if $\left\{S_{n} ; n \geqslant 1\right\}=\left\{X_{1}+\cdots+X_{n} ; n \geqslant 1\right\}$ is a discrete time random walk which has independent and identical step distribution $F$ supported on $(-\infty, \infty)$ with negative mean, denoted by $-\mu<0$, and such that $F_{\mathrm{e}}(x) \in \mathscr{S}$, then

$$
\mathbb{P}\left\{\sup _{0 \leqslant n<\infty} S_{n}>x\right\} \sim \frac{1}{\mu} \int_{x}^{\infty} \bar{F}(y) \mathrm{d} y, \quad x \rightarrow \infty,
$$

where $S_{0}=0$ by convention.

In most applications the assumption of independent step sizes is, clearly, unrealistic. Mikosch and Samorodnitsky (2000) used a two-sided linear process to model the dependent steps of a random walk and considered the asymptotic behavior of the supremum of the random walk. Under a stronger regular variation tail condition instead of subexponentiality, they obtained an asymptotic formula which might be different from (1.4) for a large class of linear processes. Their result shows that the dependence structure sometimes has a significant influence on the tail behavior of the supremum of the random walk.

Tang $(2002,2006)$ suggested to study precise large deviations for sums of negatively associated (NA) r.v.'s. In the present paper, we use the same dependence structure as suggested by Tang (2002) to model the dependent steps. A finite family of r.v.'s $\left\{X_{i}, 1 \leqslant i \leqslant n\right\}$ is said to be NA if for every pair of disjoint subsets $A_{1}$ 
and $A_{2}$ of $\{1,2, \ldots, n\}$,

$$
\operatorname{Cov}\left\{f_{1}\left(X_{i}, i \in A_{1}\right), f_{2}\left(X_{j}, j \in A_{2}\right)\right\} \leqslant 0
$$

whenever $f_{1}$ and $f_{2}$ are two coordinatewise increasing functions such that their covariance exists. An infinite family is NA if each of its finite subfamilies is NA. This definition was introduced by Alam and Saxena (1981) and Joag-Dev and Proschan (1983). As pointed out in the cited references, the NA property is possessed by many popular multivariate distributions. It is well known that non-decreasing functions defined on disjoint subsets of a set of NA r.v.'s are also NA; see, for example, Joag-Dev and Proschan (1983).

Now one question naturally arises as whether and how much the negative association of the dependent steps influences the tail behavior of the supremum of the random walk. In the paper, for a walk with NA steps which have a common distribution such that its left tail is lighter than its right tail and its equilibrium distribution has a consistently varying tail, we prove that (1.4) remains valid under a mild moment condition.

\subsection{Framework and main result}

Form now on, we assume the following basic conditions:

(a) $X_{n}, n \geqslant 1$, constitute a sequence of NA r.v.'s with a common d.f. $F$ supported on $(-\infty, \infty)$ such that $\mathbb{E} X_{1}=-\mu<0$.

(b) Assume the following tail condition: as $x \rightarrow+\infty$,

$$
F(-x)=\mathrm{o}(\bar{F}(x)) \text {. }
$$

Now we state the main result of this paper as follows:

Theorem 1.1. Under conditions $(a)$ and $(b)$, if $F_{\mathrm{e}} \in \mathscr{C}$, then the Embrechts-Veraverbeke formula (1.4) holds.

Remark. Su and Tang (2003) showed that $F_{\mathrm{e}} \in \mathscr{D}$ if and only if $F_{\mathrm{e}} \in \mathscr{C}$. So Theorem 1.1 remains valid if $F_{\mathrm{e}} \in \mathscr{D}$.

The remaining part of this paper consists of two sections. Section 2 prepares some preliminaries related to heavy-tailed distributions, whereas the proof of Theorem 1.1 is given in Section 3, which is formulated into two subsections as the proofs of the upper and lower bounds of $\mathbb{P}\left\{\sup _{1 \leqslant n<\infty} S_{n}>x\right\}$, respectively.

\section{Preliminaries}

Lemma 2.1. Let $X$ be a non-negative r.v. with finite mean $v$ and $d . f$. $F$ such that $F_{\mathrm{e}} \in \mathscr{D}$. Then for any fixed $y>0$,

$$
\limsup _{x \rightarrow \infty} \frac{x \mathbb{P}(X>y x)}{\bar{F}_{\mathrm{e}}(x)}<\infty \text { and } \limsup _{x \rightarrow \infty} \frac{\mathbb{E} X \rrbracket_{[X>y x]}}{\bar{F}_{\mathrm{e}}(x)}<\infty .
$$

Proof. Obviously, we can get from $F_{\mathrm{e}} \in \mathscr{D}$ that for any fixed $0<y<1$,

$$
\limsup _{x \rightarrow \infty} \frac{x \bar{F}(x)}{\int_{x}^{\infty} \bar{F}(t) \mathrm{d} t} \leqslant \limsup _{x \rightarrow \infty} \frac{1}{1-y} \frac{\int_{x y}^{x} \bar{F}(t) \mathrm{d} t}{\int_{x}^{\infty} \bar{F}(t) \mathrm{d} t}=\limsup _{x \rightarrow \infty} \frac{1}{1-y}\left(\frac{\bar{F}_{\mathrm{e}}(x y)}{\bar{F}_{\mathrm{e}}(x)}-1\right)<\infty .
$$

Hence, the first conclusion in (2.1) is obtained immediately from $F_{\mathrm{e}} \in \mathscr{D}$ and

$$
\frac{x \mathbb{P}(X>y x)}{\bar{F}_{\mathrm{e}}(x)}=\frac{x \bar{F}(y x)}{\bar{F}_{\mathrm{e}}(x)}=\frac{1}{y} \times \frac{y x \bar{F}(y x)}{\bar{F}_{\mathrm{e}}(y x)} \times \frac{\bar{F}_{\mathrm{e}}(y x)}{\bar{F}_{\mathrm{e}}(x)} .
$$

Applying the integration by parts we get

$$
\mathbb{E} X \rrbracket_{[X>y x]} \leqslant y x \mathbb{P}(X>y x)+v \bar{F}_{\mathrm{e}}(y x) .
$$

This, together with the first conclusion, implies the second inequality in (2.1). 
For a d.f. $F$ and any $y>0$, as done recently by Tang and Tsitsiashvili (2003) and Wang and Tang (2006), we define

$$
\bar{F}_{*}(y)=\liminf _{x \rightarrow \infty} \frac{\bar{F}(x y)}{\bar{F}(x)} \quad \text { and } \quad \rrbracket_{F}^{+}=-\lim _{y \rightarrow \infty} \frac{\log \bar{F}_{*}(y)}{\log y} .
$$

We call $\rrbracket_{F}^{+}$the upper Matuszewska index of $F$. For details we refer to Bingham et al. 1987, Chapter 2.1, Tang and Tsitsiashvili (2003) and Wang and Tang (2006), among others.

The following lemma is a combination of Bingham et al. (1987) and Tang and Tsitsiashvili (2003, Lemma 3.5):

Lemma 2.2. For a d.f. $F \in \mathscr{D}$ with its upper Matuszewska index $\mathbb{J}_{F}^{+}$, we have,

(1) for any $p>\mathbb{J}_{F}^{+}$there exist positive constants $C$ and $D$ such that the inequality

$$
\frac{\bar{F}(y)}{\bar{F}(x)} \leqslant C\left(\frac{x}{y}\right)^{p}
$$

holds for all $x \geqslant y \geqslant D$;

(2) for any $p>\sqrt{ }_{F}^{+}, x^{-p}=\mathrm{o}(\bar{F}(x))$.

The following result is due to Tang (2002). Here we copy its proof.

Lemma 2.3. Let $\left\{U_{k}, k \geqslant 1\right\}$ be a sequence of $N A$ r.v.'s with common d.f. and zero mean value, satisfying that $\mathbb{E} \exp \left\{s_{0} U_{1}\right\}<\infty$ for some $s_{0}>0$. Then for any $\gamma>0$ there exists some constant $r_{\gamma}>0$ such that for all $n \geqslant 1$ and all $x \geqslant \gamma n$,

$$
\mathbb{P}\left(\sum_{k=1}^{n} U_{k} \geqslant x\right) \leqslant \exp \left\{-r_{\gamma} x\right\}
$$

Proof. Clearly $\mathbb{E} \exp \left\{s U_{1}\right\}<\infty$ for all $0<s<s_{0}$. If $\mathbb{E} \exp \left\{s U_{1}\right\} \leqslant 1$ for some $0<s<s_{0}$, then by the NA property of the sequence $\left\{U_{k}, k \geqslant 1\right\}$,

$$
\mathbb{P}\left(\sum_{k=1}^{n} U_{k} \geqslant x\right) \leqslant \mathrm{e}^{-s x} \mathbb{E} \exp \left\{s \sum_{k=1}^{n} U_{k}\right\} \leqslant \mathrm{e}^{-s x} \mathbb{E}^{n} \exp \left\{s U_{1}\right\} \leqslant \mathrm{e}^{-s x},
$$

which means that (2.3) holds with $r_{\gamma}=s$. If $\mathbb{E} \exp \left\{s U_{1}\right\}>1$ for all $0<s<s_{0}$, then by the NA property of the sequence $\left\{U_{k}, k \geqslant 1\right\}$ we can get that for any $0<s<s_{0}$ and $x \geqslant \gamma n$,

$$
\mathbb{P}\left(\sum_{k=1}^{n} U_{k} \geqslant x\right) \leqslant \exp \left\{n \log \mathbb{E} \exp \left\{s U_{1}\right\}-s x\right\} \leqslant \exp \left\{x\left(\frac{1}{\gamma} \log \mathbb{E} \exp \left\{s U_{1}\right\}-s\right)\right\} .
$$

Write $f(s)=(1 / \gamma) \log \mathbb{E} \exp \left\{s U_{1}\right\}-s$ for $0<s<s_{0}$, which is differentiable for any times in $s \in\left[0, s_{0}\right)$ and satisfies $f(0)=0, f^{\prime}(0)=-1$. Furthermore, by the classical Hölder's inequality we obtain

$$
f^{\prime \prime}(s)=\frac{\mathbb{E} U_{1}^{2} \exp \left\{s U_{1}\right\} \cdot \mathbb{E} \exp \left\{s U_{1}\right\}-\left(\mathbb{E} U_{1} \exp \left\{s U_{1}\right\}\right)^{2}}{\gamma\left(\mathbb{E} \exp \left\{s U_{1}\right\}\right)^{2}} \geqslant 0 \text { for } 0<s<s_{0} .
$$

So we can find some $s_{\gamma} \in\left(0, s_{0}\right)$ such that the (optimal) value $f\left(s_{\gamma}\right)<0$, which gives the inequality (2.3) with $r_{\gamma}=-f\left(s_{\gamma}\right)>0$. It ends the proof of the lemma.

Lemma 2.4. Let $U_{n}, n \geqslant 1$, constitute a sequence of $N A$ r.v.'s with zero mean and common d.f. F supported on $(-\infty, \infty)$. Suppose that there exists a d.f. $G$ with $G_{\mathrm{e}} \in \mathscr{D}$ such that $\bar{F} \lesssim \bar{G}$. Then, for any fixed $u>0$ and $1>\theta>0$

$$
\mathbb{P}\left\{\bigcup_{n \geqslant 1}\left(\sum_{j=1}^{n} U_{j}>x+n u\right), \bigcap_{i \geqslant 1}\left(U_{i} \leqslant \theta(i u+x)\right)\right\}=\mathrm{o}\left(\bar{G}_{\mathrm{e}}(x)\right) .
$$


Proof. We adopt the way suggested by Tang (2002) with great changes. Take $0<\delta<(1-\theta) / 3$ and $\alpha>1$ such that $\theta \alpha<1-3 \delta$. Let $K>0$ satisfy $\mathbb{E} U_{1} \rrbracket_{\left[U_{1} \geqslant K\right]}<u \delta / 2 \alpha$. Write

$$
\dot{U}_{n k}=U_{k} \rrbracket_{\left[K \leqslant U_{k} \leqslant \theta(n u+x)\right]}+\theta(n u+x) \rrbracket_{\left[U_{k}>\theta(n u+x)\right]}, k, n \geqslant 1, \quad \ddot{U}_{k}=U_{k} \rrbracket_{\left[U_{k}<K\right]}+K \rrbracket_{\left[U_{k} \geqslant K\right]}, k \geqslant 1 .
$$

Clearly, $0=-\mathbb{E} U_{j} \leqslant-\mathbb{E} \ddot{U}_{j}$ for all $j \geqslant 1$. Hence we can see that for large enough $x$,

$$
\begin{aligned}
& \mathbb{P}\left\{\bigcup_{n \geqslant 1}\left(\sum_{j=1}^{n} U_{j}>x+n u\right), \bigcap_{i \geqslant 1}\left(U_{i} \leqslant \theta(i u+x)\right)\right\} \\
& \quad \leqslant \mathbb{P}\left\{\bigcup_{n \geqslant 1}\left(\sum_{j=1}^{n} \dot{U}_{n j}>(1-\delta)(x+n u)\right)\right\}+\mathbb{P}\left\{\bigcup_{n \geqslant 1}\left(\sum_{j=1}^{n}\left(\ddot{U}_{j}-\mathbb{E}_{j}\right)>\delta(x+n u)\right)\right\}=I_{1}+I_{2} .
\end{aligned}
$$

Notice that $\mathbb{E} \dot{U}_{n k} \leqslant \mathbb{E} U_{1} \square_{\left[U_{1} \geqslant k\right]}<u \delta / 2 \alpha$ for all $k, n \geqslant 1$ and that for large enough $x$, $x+\left[\alpha^{i-1}\right] u \geqslant x+\left(\alpha^{i-1}-1\right) u \geqslant \alpha^{-1}\left(x+\left[\alpha^{i}\right] u\right)-u \geqslant \alpha^{-1}\left(x+\left[\alpha^{i}\right] u\right)\left(\left(1-\frac{3}{2} \delta\right) /(1-\delta)\right)$. Hence we derive, for large enough $x>0$ and any fixed $h_{i}=h(x, i)>0$,

$$
\begin{aligned}
I_{1} & \leqslant \sum_{i=1}^{\infty} \mathbb{P}\left\{\sum_{j=1}^{\left[\alpha^{i}\right]} \dot{U}_{\left[\alpha^{i}\right] j}>\left(x+\left[\alpha^{i-1}\right] u\right)(1-\delta)\right\} \\
& \leqslant \sum_{i=1}^{\infty} \mathbb{P}\left\{\sum_{j=1}^{\left[\alpha^{i}\right]}\left(\dot{U}_{\left[\alpha^{i}\right] j}-\mathbb{E} \dot{U}_{\left[\alpha^{i}\right] j}\right)>\alpha^{-1}\left(x+\left[\alpha^{i}\right] u\right)(1-2 \delta)\right\} \\
& \leqslant \sum_{i=1}^{\infty} \mathbb{E}^{\left[\alpha^{i}\right]} \exp \left\{h_{i} \dot{U}_{\left[\alpha^{i}\right] 1}\right\} \exp \left\{-h_{i} \alpha^{-1}\left(x+\left[\alpha^{i}\right] u\right)(1-2 \delta)-h_{i}\left[\alpha^{i}\right] \mathbb{E} \dot{U}_{\left[\alpha^{i}\right] 1}\right\},
\end{aligned}
$$

where in the last inequality we used the fact that for any fixed $i,\left\{\dot{U}_{[\alpha i] k}, k \geqslant 1\right\}$ is still a sequence of NA r.v.'s with common d.f. Denote $b(x, i)=\theta\left(x+\left[\alpha^{i}\right] u\right)$ and $a_{i}=a_{i}(x)=-\log \left(\left[\alpha^{i}\right] \bar{G}(b(x, i))\right)$. Clearly, $a_{i} \rightarrow \infty$ as $x \rightarrow \infty$. Write $\mu_{\left[\alpha^{i}\right]}=\mathbb{E} \dot{U}_{\left[\alpha^{i}\right] 1}$ and introduce

$$
f_{i}=\left[\alpha^{i}\right] \mathrm{e}^{h_{i} b(x, i)} \bar{F}(b(x, i))+\left[\alpha^{i}\right]\left(\int_{K}^{b(x, i) / a_{i}^{\gamma}}+\int_{b(x, i) / a_{i}^{\gamma}}^{b(x, i)}\right)\left(\mathrm{e}^{h_{i} t}-1\right) F(\mathrm{~d} t)-h_{i}\left[\alpha^{i}\right] \mu_{\left[\alpha^{i}\right]},
$$

where $\gamma>1$ is a fixed constant. Hence we have

$$
\begin{aligned}
I_{1} \leqslant & \sum_{i=1}^{\infty}\left(\int_{K}^{b(x, i)}\left(\mathrm{e}^{h_{i} t}-1\right) F(\mathrm{~d} t)+\mathrm{e}^{h_{i} b(x, i)} \bar{F}(b(x, i))+1\right)^{\left[\alpha^{i}\right]} \\
& \times \exp \left\{-h_{i} \alpha^{-1}\left(x+\left[\alpha^{i}\right] u\right)(1-2 \delta)-h_{i}\left[\alpha^{i}\right] \mu_{\left[\alpha^{i}\right]}\right\} \\
\leqslant & \sum_{i=1}^{\infty} \exp \left\{f_{i}-h_{i} \alpha^{-1}\left(x+\left[\alpha^{i}\right] u\right)(1-2 \delta)\right\} .
\end{aligned}
$$

Applying an elementary inequality that $\mathrm{e}^{s}-1 \leqslant s \mathrm{e}^{s}$ for $s>0$, we can obtain that for large enough $x$, the first integral in $f_{i}$ is bounded from above by

$$
\int_{K}^{b(x, i) / a_{i}^{\gamma}}\left(\mathrm{e}^{h_{i} t}-1\right) F(\mathrm{~d} t) \leqslant \exp \left\{\frac{h_{i} b(x, i)}{a_{i}^{\gamma}}\right\} \int_{K}^{b(x, i) / a_{i}^{\gamma}} h_{i} t F(\mathrm{~d} t) \leqslant h_{i} \mu_{\left[\alpha^{i}\right]} \exp \left\{\frac{h_{i} b(x, i)}{a_{i}^{\gamma}}\right\} .
$$

As for the second integral there, using Lemma 2.2 and $\bar{F} \lesssim \bar{G}$ we derive, for large enough $x$,

$$
\int_{b(x, i) / a_{i}^{\gamma}}^{b(x, i)}\left(\mathrm{e}^{h_{i} t}-1\right) F(\mathrm{~d} t) \leqslant C \bar{G}\left(\frac{b(x, i)}{a_{i}^{\gamma}}\right) \exp \left\{h_{i} b(x, i)\right\} \leqslant C a_{i}^{\gamma p} \bar{G}(b(x, i)) \exp \left\{h_{i} b(x, i)\right\},
$$


where $p>\mathbb{J}_{F}^{+}$. Plugging (2.6) and (2.7) into the expression of $f_{i}$ and Noting $\bar{F} \lesssim \bar{G}$ yield that

$$
f_{i} \leqslant C\left[\alpha^{i}\right] \mathrm{e}^{h_{i} b(x, i)} \bar{G}(b(x, i))\left(1+a_{i}^{\gamma p}\right)+\left[\alpha^{i}\right]\left(\exp \left\{\frac{h_{i} b(x, i)}{a_{i}^{\gamma}}\right\}-1\right) h_{i} \mu_{\left[\alpha^{i}\right]}
$$

holds for large enough $x$. Let $h_{i}=\left(a_{i}-\gamma p \log a_{i}\right) b^{-1}(x, i)$. Notice that $h_{i} b(x, i)=\mathrm{o}\left(a_{i}^{\gamma}\right)$ holds uniformly for $i \geqslant 1,0 \leqslant \mu_{\left[\alpha^{i}\right]} \leqslant \mathbb{E} U_{1} \square_{\left[U_{1} \geqslant K\right]}<u \delta / 2 \alpha$ for all $i \geqslant 1$ and $\left[\alpha^{i}\right] h_{i} \leqslant h_{i} b(x, i) / u \theta$. Hence,

$$
f_{i}-h_{i} \alpha^{-1}\left(x+\left[\alpha^{i}\right] u\right)(1-2 \delta) \leqslant C+\mathrm{o}\left(a_{i}\right)-\frac{\left(a_{i}-\gamma p \log a_{i}\right)(1-2 \delta)}{\theta \alpha} \leqslant-\frac{(1-3 \delta) a_{i}}{\theta \alpha}
$$

holds for all large $x$. Notice that $\left[\alpha^{i}\right] \bar{G}\left(\theta\left(\left[\alpha^{i}\right] u+x\right)\right) \leqslant C \int_{\theta\left(x+\left[\alpha^{i-1}\right] u\right)}^{\theta\left(x+\left[\alpha^{i}\right] u\right)} \bar{G}(t) \mathrm{d} t$ for large enough $i$ and $\left[\alpha^{i}\right] \bar{G}\left(\theta\left(\left[\alpha^{i}\right] u+\right.\right.$ $x)) \leqslant C \int_{\theta x}^{\infty} \bar{G}(t) \mathrm{d} t$ for all $i \geqslant 1$. Hence, substituting the above inequalities into (2.5) and in view of $\theta \alpha<1-3 \delta$ and $G_{\mathrm{e}} \in \mathscr{D}$ we conclude that for large enough $x$,

$$
I_{1} \leqslant \sum_{i=1}^{\infty}\left(\left[\alpha^{i}\right] \bar{G}\left(\theta\left(\left[\alpha^{i}\right] u+x\right)\right)\right)^{(1-3 \delta) / \theta \alpha} \leqslant C\left(\int_{\theta x}^{\infty} \bar{G}(t) \mathrm{d} t\right)^{((1-3 \delta) / \theta \alpha)-1} \int_{\theta x}^{\infty} \bar{G}(t) \mathrm{d} t=\mathrm{o}\left(\bar{G}_{\mathrm{e}}(x)\right) .
$$

As for $I_{2}$, using Lemma 2.3 we have

$$
I_{2} \leqslant \sum_{n=1}^{\infty} \exp \left\{-r_{\gamma} \delta(x+n u)\right\}=\mathrm{o}\left(\bar{G}_{\mathrm{e}}(x)\right)
$$

where in the last step we used Lemma 2.2. Plugging (2.9) and (2.10) into (2.4) we get the Lemma.

\section{Proof of the main result}

In this section we will use a generic r.v. $X$ with the same distribution as $X_{1}$. Let $0<\delta<\frac{1}{3}$ be small enough and $\theta$ be any fixed positive number. For simplicity we introduce the following notations:

$$
\begin{aligned}
& A(x)=\left\{\sup _{n \geqslant 1} S_{n}>x\right\}=\left\{\sup _{n \geqslant 1}\left(-\mu n+\sum_{j=1}^{n}\left(X_{j}-\mathbb{E} X_{j}\right)\right)>x\right\}, \\
& A_{1}(x, \delta)=\left\{A(x) ; \bigcup_{j \geqslant 1}\left(X_{j}>(1-\delta)(j \mu+x)\right)\right\}, \\
& A_{2}(x, \delta)=\left\{A(x), \bigcap_{i \geqslant 1}\left(X_{i}-\mathbb{E} X_{i} \leqslant \theta(i \mu+x)\right)\right\}, \\
& A_{3}(x, \delta)=\left\{A(x), \bigcap_{j \geqslant 1}\left(X_{j} \leqslant(1-\delta)(j \mu+x)\right), \bigcup_{i \geqslant 1}\left(X_{i}-\mathbb{E} X_{i}>\theta(i \mu+x)\right)\right\} .
\end{aligned}
$$

Now we formulate the proof of Theorem 1.1 into two parts, which provide the upper and lower bounds for $\mathbb{P}\left\{\sup _{n \geqslant 1} S_{n}>x\right\}$, respectively.

\subsection{Upper bound: $\mathbb{P}\{A(x)\} \lesssim \mu^{-1} \int_{x}^{\infty} \bar{F}(x) \mathrm{d} x$}

Clearly we have

$$
\mathbb{P}\left\{\sup _{n \geqslant 1} S_{n}>x\right\} \leqslant \mathbb{P}\left\{A_{1}(x, \delta)\right\}+\mathbb{P}\left\{A_{2}(x, \delta)\right\}+\mathbb{P}\left\{A_{3}(x, \delta)\right\} .
$$

Using Lemma 2.4 we conclude that

$$
\mathbb{P}\left\{A_{2}(x, \delta)\right\} \leqslant \mathbb{P}\left\{A(x) ; \bigcap_{i \geqslant 1}\left(X_{i}-\mathbb{E} X_{i} \leqslant \theta(i \mu+x)\right)\right\}=\mathrm{o}\left(\bar{F}_{\mathrm{e}}(x)\right) .
$$


Next we deal with $\mathbb{P}\left\{A_{1}(x, \delta)\right\}$. To save notations, we denote $b_{1}(l)=b_{1}(l, x, \delta)=(1-\delta)(l \mu+x)$. Noticing that $\left\{X_{j} ; j \geqslant 1\right\}$ are i.i.d., we obtain

$$
\begin{aligned}
\mathbb{P}\left\{A_{1}(x, \delta)\right\} & \leqslant \sum_{j=1}^{\infty} \sum_{l=j}^{\infty} \mathbb{P}\left\{b_{1}(l+1) \geqslant X>b_{1}(l)\right\} \\
& =\sum_{l=1}^{\infty} \frac{l \mu+x}{\mu} \mathbb{P}\left\{b_{1}(l+1) \geqslant X>b_{1}(l)\right\}-\sum_{l=1}^{\infty} \frac{x}{\mu} \mathbb{P}\left\{b_{1}(l+1) \geqslant X>b_{1}(l)\right\} \\
& \leqslant \frac{1}{\mu(1-\delta)} \mathbb{E} X \rrbracket_{\left[X>b_{1}(1)\right]}-\frac{x}{\mu} \mathbb{P}\left\{X>b_{1}(1)\right\} \\
& \leqslant \mathbb{P}\left\{X>b_{1}(1)\right\}+\frac{1}{\mu(1-\delta)} \int_{b_{1}(1)}^{\infty} \mathbb{P}(X>x) \mathrm{d} x
\end{aligned}
$$

where in the last inequality we used the integration by parts. Notice $\mathscr{C} \subset \mathscr{D}$. Hence, by Lemma 2.1 and $F_{\mathrm{e}} \in \mathscr{C}$ we can get that

$$
\lim _{\delta \searrow 0} \limsup _{x \rightarrow \infty} \frac{\mathbb{P}\left\{A_{1}(x, \delta)\right\}}{(1 / \mu) \int_{x}^{\infty} \bar{F}(x) d x} \leqslant \lim _{\delta \searrow 0} \limsup _{x \rightarrow \infty} \frac{\bar{F}_{\mathrm{e}}\left(b_{1}(1)\right)}{\bar{F}_{\mathrm{e}}(x)}=1 .
$$

Hence, in order to complete the proof of this part, it suffices to prove that the term $\mathbb{P}\left\{A_{3}(x, \delta)\right\}$ is asymptotically negligible as $x \rightarrow \infty$ when compared with $\bar{F}_{\mathrm{e}}(x)$. Notice $0<\delta<\frac{1}{3}$ and $\left\{X_{l} \leqslant b_{1}(l)\right\} \subset\left\{X_{l} \leqslant b_{1}(n)\right\}$ for all $l \leqslant n$. Hence we can get that for large enough $x$,

$$
\left\{A(x), \bigcap_{j \geqslant 1}\left(X_{j} \leqslant b_{1}(j)\right), X_{l}-\mathbb{E} X_{l}>\theta(l \mu+x)\right\} \subset\left\{\bigcup_{n \geqslant 1} B_{n l}, X_{l}-\mathbb{E} X_{l}>\theta(l \mu+x)\right\},
$$

where

$$
B_{n l}=\left\{\sum_{i=1, i \neq l}^{n}\left(X_{i}-\mathbb{E} X_{i}\right)>\delta(n \mu+x)+\mathbb{E} X_{l}\right\} .
$$

By convention $\sum_{i=1, i \neq l}^{n}=\sum_{i=1}^{n}$ for $n<l$. We can get from NA property that for large $x$ and any $\theta_{1}>0$,

$$
\begin{aligned}
\mathbb{P}\left\{A_{3}(x, \delta)\right\} \leqslant & \sum_{l=1}^{\infty} \mathbb{P}\left\{\bigcup_{n=1}^{\infty} B_{n l}\right\} \mathbb{P}\left\{X_{l}-\mathbb{E} X_{l}>\theta(l \mu+x)\right\} \\
\leqslant & \sum_{l=1}^{\infty} \sum_{j=1}^{\infty} \mathbb{P}\left\{X_{j}-\mathbb{E} X_{j}>\theta_{1}(j \mu+x)\right\} \mathbb{P}\left\{X_{l}>\theta_{2}(l \mu+x)\right\} \\
& +\sum_{l=1}^{\infty} \mathbb{P}\left\{\bigcup_{n=1}^{\infty} B_{n l}, \bigcap_{j \geqslant 1}\left(X_{j}-\mathbb{E} X_{j} \leqslant \theta_{1}(j \mu+x)\right)\right\} \mathbb{P}\left\{X_{l}>\theta_{2}(l \mu+x)\right\} \\
= & I_{1}+I_{2},
\end{aligned}
$$

where $0<\theta_{2}<\theta$. Clearly we can get that for large enough $x$ and any $0<\theta_{0}<\min \left\{\theta_{1}, \theta_{2}\right\}$,

$$
\sum_{l=1}^{\infty} \mathbb{P}\left\{X_{l}>\theta_{0}(l \mu+x)\right\} \leqslant \sum_{k=1}^{\infty} k \mathbb{P}\left\{\theta_{0}((k+1) \mu+x) \geqslant X>\theta_{0}(k \mu+x)\right\} \leqslant \frac{1}{\theta_{0} \mu} \mathbb{E} X \rrbracket_{\left[X>\theta_{0} x\right]} .
$$

Therefore, using Lemma 2.1 we can get

$$
I_{1} \leqslant\left(\sum_{j=1}^{\infty} \mathbb{P}\left\{X_{j}>\theta_{0}(j \mu+x)\right\}\right)^{2}=\mathrm{o}\left(\bar{F}_{\mathrm{e}}(x)\right) .
$$


Similarly to the proof of (3.2), we can get from Lemma 2.4 and (3.5) that for some $0<\delta_{0}<\delta$,

$$
I_{2} \leqslant \sum_{l=1}^{\infty} \mathbb{P}\left\{\bigcup_{n=1}^{\infty} C_{n l}, \bigcap_{j \geqslant 1}\left(X_{j}-\mathbb{E} X_{j}>\theta_{1}(j \mu+x)\right)\right\} \mathbb{P}\left\{X_{l}>\theta_{2}(l \mu+x)\right\}=\mathrm{o}\left(\bar{F}_{\mathrm{e}}(x)\right),
$$

where

$$
C_{n l}=\left\{\sum_{i=1, i \neq l}^{n}\left(X_{i}-\mathbb{E} X_{i}\right)>\delta_{0}(n \mu+x)\right\} .
$$

Substituting (3.6) and (3.7) into (3.4) yields

$$
\mathbb{P}\left\{A_{3}(x, \delta)\right\}=\mathrm{o}\left(\bar{F}_{\mathrm{e}}(x)\right) .
$$

This ends the proof of Theorem 1.1 corresponding to the upper bound.

3.2. Lower bound: $\mathbb{P}\{A(x)\} \gtrsim \mu^{-1} \int_{x}^{\infty} \bar{F}(x) \mathrm{d} x$

Denote $b_{2}(j)=b_{2}(j, x, \delta)=(1+\delta)(j \mu+x)$. For the sake of simplicity, we write

$$
B=\bigcap_{j>1}\left(\sum_{l=1}^{j-1}\left(X_{l}-\mathbb{E} X_{l}\right) \geqslant-\delta(j \mu+x)+\mathbb{E} X\right) \quad \text { and } \quad L=\inf \left\{j: X_{j}>b_{2}(j)\right\} .
$$

Hence we have

$$
\begin{aligned}
\mathbb{P}\{A(x)\} & \geqslant \sum_{j=2}^{\infty} \mathbb{P}\left\{\sum_{l=1}^{j}\left(X_{l}-\mathbb{E} X_{l}\right)>j \mu+x, L=j\right\} \\
& \geqslant \sum_{j=2}^{\infty} \mathbb{P}\{B, L=j\} \\
& \geqslant \sum_{j=2}^{\infty} \mathbb{P}\{L=j\}-\sum_{j=2}^{\infty} \mathbb{P}\left\{B^{c}, L=j\right\} \\
& \geqslant \sum_{j=2}^{\infty} \mathbb{P}\left\{\bigcap_{1 \leqslant k<j}\left(X_{k} \leqslant b_{2}(k)\right), X_{j}>b_{2}(j)\right\}-\mathbb{P}\left\{B^{c}\right\} \\
& =\sum_{j=2}^{\infty} \mathbb{P}\left\{X_{j}>b_{2}(j)\right\}-\sum_{j=2}^{\infty} \mathbb{P}\left\{\bigcup_{1 \leqslant k<j}\left(X_{k}>b_{2}(k)\right), X_{j}>b_{2}(j)\right\}-\mathbb{P}\left\{B^{c}\right\} \\
& \geqslant \sum_{j=2}^{\infty} \mathbb{P}\left\{X_{j}>b_{2}(j)\right\}-\sum_{j=2}^{\infty} \sum_{k=1}^{j-1} \mathbb{P}\left\{X_{k}>b_{2}(k)\right\} \mathbb{P}\left\{X_{j}>b_{2}(j)\right\}-\mathbb{P}\left\{B^{c}\right\},
\end{aligned}
$$

where in the last step we used the assumption that $\left\{X_{k} ; k \geqslant 1\right\}$ is a sequence of NA r.v.'s. At first we consider $\sum_{j=2}^{\infty} \mathbb{P}\left\{X_{j}>b_{2}(j)\right\}$. We can see that

$$
\begin{aligned}
\sum_{j=2}^{\infty} \mathbb{P}\left\{X_{j}>b_{2}(j)\right\} & =\sum_{j=2}^{\infty} \sum_{l=j}^{\infty} \mathbb{P}\left\{b_{2}(l+1) \geqslant X>b_{2}(l)\right\} \\
& =\sum_{l=2}^{\infty} \frac{(l+1) \mu+x}{\mu} \mathbb{P}\left\{b_{2}(l+1) \geqslant X>b_{2}(l)\right\}-\left(\frac{x}{\mu}+2\right) \mathbb{P}\left\{X>b_{2}(2)\right\} \\
& \geqslant \frac{1}{(1+\delta) \mu} \mathbb{E} X \rrbracket_{[X>(1+\delta)(2 \mu+x)]}-\left(\frac{x}{\mu}+2\right) \mathbb{P}\{X>(1+\delta)(2 \mu+x)\} \\
& =\frac{1}{(1+\delta) \mu} \int_{(1+\delta)(2 \mu+x)}^{\infty} \mathbb{P}(X>x) d x
\end{aligned}
$$


Notice that $F_{\mathrm{e}} \in \mathscr{C}$. Hence we can derive

$$
\lim _{\delta \searrow 0} \liminf _{x \rightarrow \infty} \frac{\sum_{j=2}^{\infty} \mathbb{P}\left\{X_{j}>b_{2}(j)\right\}}{\mu^{-1} \int_{x}^{\infty} \bar{F}(x) d x} \geqslant \lim _{\delta \searrow 0} \liminf _{x \rightarrow \infty} \frac{\bar{F}((1+\delta)(2 \mu+x))}{\bar{F}_{\mathrm{e}}(x)}=1 .
$$

Using (3.5) with $\theta_{0}=1+\delta$ and Lemma 2.1 we can see

$$
\sum_{j=2}^{\infty} \sum_{k=1}^{j-1} \mathbb{P}\left\{X_{k}>b_{2}(k)\right\} \mathbb{P}\left\{X_{j}>b_{2}(j)\right\} \leqslant\left(\sum_{j=1}^{\infty} \mathbb{P}\left\{X_{j}>b_{2}(j)\right\}\right)^{2}=\mathrm{o}\left(\bar{F}_{\mathrm{e}}(x)\right) .
$$

Hence, in order to end the part, it suffices to show that the third term in (3.9) is asymptotically negligible when compared with $\bar{F}_{\mathrm{e}}(x)$. Let us denote $U_{j}=-X_{j}+\mathbb{E} X_{j}, j=1,2, \ldots, x_{j}=\delta_{1}(x+j \mu)$ for $j=1,2, \ldots$, where $0<\delta_{1}<\delta$. Clearly, $\delta(j \mu+x)-\mathbb{E} X \geqslant \delta_{1}(j \mu+x)$ for large $x$. Hence, for any fixed $\theta_{3}>0$ and large enough $x$ we have

$$
\begin{aligned}
\mathbb{P}\left\{B^{c}\right\} & \leqslant \mathbb{P}\left\{\bigcup_{j>1}\left(\sum_{l=1}^{j-1} U_{l} \geqslant x_{j}\right)\right\} \\
& \leqslant \sum_{j=1}^{\infty} \mathbb{P}\left\{U_{j}>\theta_{3} x_{j}\right\}+\mathbb{P}\left\{\bigcup_{j>1}\left(\sum_{l=1}^{j-1} U_{l} \geqslant x_{j}\right), \bigcap_{j \geqslant 1}\left[U_{j} \leqslant \theta_{3} x_{j}\right]\right\} .
\end{aligned}
$$

Noticing condition b) we see that $\left\{U_{j} ; j=1,2, \ldots\right\}$ have a common d.f. $H$ such that $\bar{H}(x)=\mathrm{o}(\bar{F}(x-\mathbb{E} X))$. Hence, we can also get from Lemma 2.4 that for fixed but small enough $\theta_{3}$,

$$
\mathbb{P}\left\{\bigcup_{j>1}\left(\sum_{l=1}^{j-1} U_{l} \geqslant x_{j}\right), \bigcap_{j \geqslant 1}\left[U_{j} \leqslant \theta_{3} x_{j}\right]\right\}=\mathrm{o}\left(\bar{F}_{\mathrm{e}}(x)\right) .
$$

Similarly to the proof of (3.5) we can get form $\mathbb{E} X_{j}<0$ that

$$
\sum_{j=1}^{\infty} \mathbb{P}\left\{U_{j} \geqslant \theta_{3} x_{j}\right\} \leqslant \sum_{j=1}^{\infty} \mathbb{P}\left\{-X_{j} \geqslant \theta_{3} x_{j}\right\} \leqslant \frac{1}{\theta_{3} \delta_{1} \mu} \mathbb{E}(-X) \rrbracket_{\left[-X>\theta_{3} \delta_{1} x\right]} .
$$

Using the integration by parts we have

$$
\begin{aligned}
\sum_{j=1}^{\infty} \mathbb{P}\left\{U_{j} \geqslant \theta_{3} x_{j}\right\} & \leqslant \frac{x}{\mu} F\left(-\theta_{3} \delta_{1} x\right)+\frac{1}{\theta_{3} \delta_{1} \mu} \int_{-\infty}^{-\theta_{3} \delta_{1} x} F(t) d t \\
& \leqslant C\left(\int_{-\infty}^{-\theta_{3} \delta_{1} x / 2} F(t) d t+\int_{-\infty}^{-\theta_{3} \delta_{1} x} F(t) d t\right) \\
& =\int_{\theta_{3} \delta_{1} x / 2}^{\infty} F(-t) d t+\int_{\theta_{3} \delta_{1} x}^{\infty} F(-t) d t=\mathrm{o}\left(\bar{F}_{\mathrm{e}}(x)\right),
\end{aligned}
$$

where in the last step we used condition (b) and $F_{\mathrm{e}} \in \mathscr{C} \subset \mathscr{D}$. This ends the part. Therefore, we complete the proof of Theorem 1.1.

\section{Acknowledgment}

As his current postdoc, Dingcheng Wang thanks professor Chris Heyde for his help and support. The authors also thank Dr. Qihe Tang for his help.

\section{References}

Alam, K., Saxena, K.M.L., 1981. Positive dependence in multivariate distributions. Comm. Statist. A Theory Methods 10, $1183-1196$. Bingham, N.H., Goldie, C.M., Teugels, J.L., 1987. Regular Variation. Cambridge University Press, Cambridge. 
Embrechts, P., Veraverbeke, N., 1982. Estimates for the probability of ruin with special emphasis on the possibility of large claims Insurance Math. Econom. 1 (1), 55-72.

Embrechts, P., Klüppelberg, C., Mikosch, T., 1997. Modelling Extremal Events for Insurance and Finance. Springer, Berlin.

Joag-Dev, K., Proschan, F., 1983. Negative association of random variables with applications. Ann. Statist. 11, $286-295$.

Mikosch, T., Samorodnitsky, G., 2000. The supremum of a negative drift random walk with dependent heavy-tailed steps. Ann. Appl. Probab. 10 (3), 1025-1064.

Su, C., Tang, Q., 2003. Characterizations on heavy-tailed distributions by means of hazard rate. Acta. Math. Appl. Sinica (English Series) 19 (1), 135-142.

Tang, Q., 2002. Large deviation probabilities for sums of negatively associated risks with heavy tails. Personal communication.

Tang, Q., 2006. Insensitivity to negative dependence of the asymptotic behavior of precise large deviations. Electron. J. Probab. 11 (4), 107-120.

Tang, Q., Tsitsiashvili, G., 2003. Precise estimates for the ruin probability in finite horizon in a discrete-time model with heavy-tailed insurance and financial risks. Stochastic Process. Appl. 108 (2), 299-325.

Wang, D.C., Tang, Q., 2006. Tail probabilities of randomly weighted sums of random variables with dominated variation. Stochastic Models 22 (2), 253-272. 